\title{
Une erreur féconde du mathématicien Henri Poincaré. Le prix en l'honneur des 60 ans du roi Oscar et la découverte des orbites homoclines
}

\author{
Jean-Christophe Yoccoz
}

Published online: 14 June 2013

(c) Centro P.RI.ST.EM, Università Commerciale Luigi Bocconi 2013

\begin{abstract}
This paper describes Poincaré's discoveries in nonlinear dynamics. The famous "error" contained in the first version of the memoir "Sur le problème des trois corps et les équations de la dynamique" is discussed.
\end{abstract}

Keywords Henri Poincaré · Three-body problem - Chaos theory $\cdot$ Celestial mechanics

«... For, in respect to the latter branch of the supposition, it should be considered that the most trifling variation in the facts of the two cases might give rise to the most important miscalculations, by diverting thoroughly the two courses of events, very much as, in arithmetic, an error which, in its own individuality, may be inappreciable, produces, at length, by dint of multiplication at all points of the process, a result enormously at variance with truth. ...»

Edgar Allan POE

'The Mystery of Marie Roget', 1843

«... Car, relativement à la dernière partie de la supposition, on doit considérer que la plus légère variation dans les éléments des deux problèmes pourrait engendrer les plus graves erreurs de calcul, en faisant diverger absolument les deux courants d'évènements; à peu près de la même manière qu'en arithmétique une erreur qui, prise individuellement, peut être inappréciable, produit à la longue, par la

J.-C. Yoccoz $(\bowtie)$

Collège de France, 11, place Marcelin Berthelot, 75231 Paris

Cedex 05, France

e-mail: jean-c.yoccoz@college-de-france.fr force accumulative de la multiplication, un résultat effroyablement distant de la vérité. ...»

Fr. Trad. Charles Baudelaire, 1864

La citation précédente est sans doute une des premières descriptions de ce qui a, beaucoup plus récemment, été baptisé d' «effet papillon», l'idée qu'à cause du caractère instable des évolutions dynamiques associées au système météorologique, le battement d'ailes d'un papillon pourrait sur le long terme être à l'origine de tempêtes et autres cataclysmes. Dans la bouche du chevalier Dupin, c'est à la logique d'une enquête policière plutôt qu'à la météorologie qu'est associée ce phénomène.

Le héros de notre histoire, Henri Poincaré (Fig. 1) naît à Nancy, 11 ans après la parution de la nouvelle de Poe. Reçu premier à l'Ecole Polytechnique, il soutient en 1879 une thèse dont une des parties, le «Mémoire sur les propriétés des fonctions définies par les équations différentielles», annonce une des directions que prendront ses recherches. Après un bref passage à Caen, il est de retour à Paris dès 1881 et occupera à la Sorbonne à partir de 1886 une chaire de « Physique mathématique et Calcul des probabilités ».

Henri Poincaré est le plus grand mathématicien de son temps, l'un des 4 ou 5 plus importants de tous les temps. Son oeuvre s'étend aussi à la physique. Avec Lorentz et Einstein, il est le codécouvreur de la théorie de la relativité restreinte. Par ailleurs, ses textes de philosophie des sciences exercent encore aujourd'hui une influence considérable. Son oeuvre proprement mathématique est immense, de la géométrie à l'analyse et la topologie. Il est aussi le fondateur de la théorie des systèmes dynamiques; c'est à cette partie de ses travaux que se rattache l'épisode qui nous intéresse ici.

Stockholm est certainement l'une des plus belles villes du monde, tout particulièrement au printemps où l'éclosion 


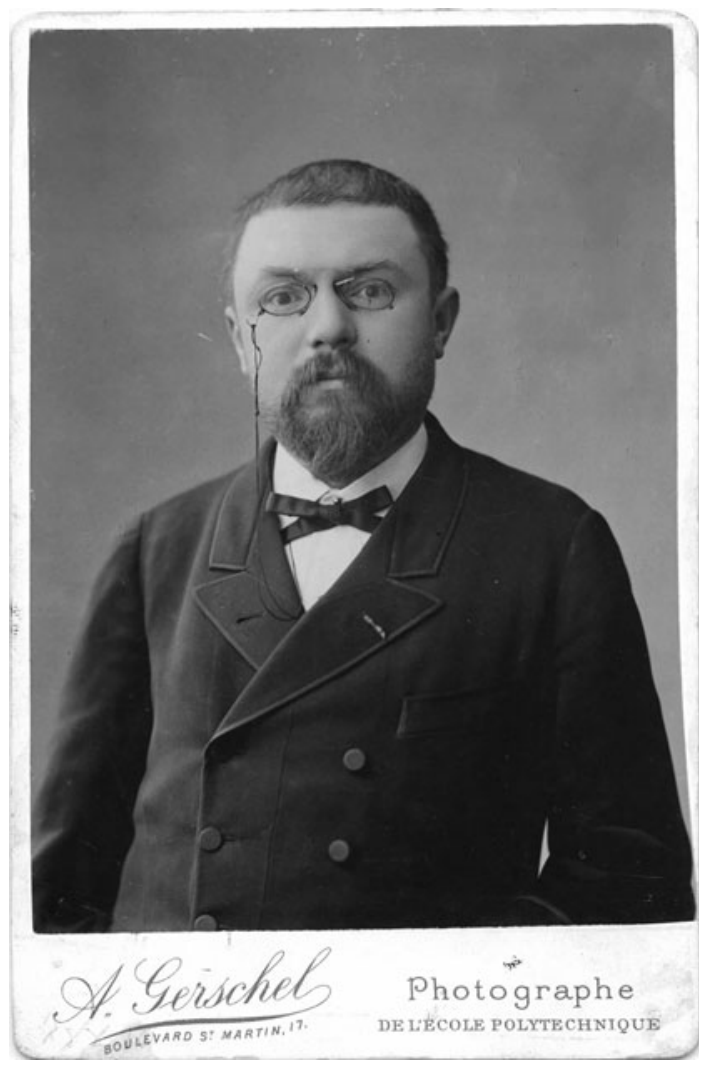

Fig. 1 Henri Poincaré (1854-1912). Reproduced by generous permission of the LHSP-Archives Henri Poincaré. UMR 7117 CNRS/Université de Lorraine

de la nature et la mer partout présente y créent une atmosphère exceptionnelle. A quelques kilomètres du centre, Djürsholm abrite au bord d'un bras de mer de splendides résidences, dont l'Institut Mittag-Leffler. Cet institut, avec la superbe bibliothèque autour duquel il s'organise, était il y a un siècle la demeure de Gösta Mittag-Leffler (Fig. 2), le second personnage de notre histoire. C'est aujourd'hui l'un des hauts lieux de la recherche mathématique en Europe.

Mittag-Leffler fut un mathématicien de tout premier ordre, spécialiste d'analyse complexe, disputant avec le chimiste Alfred Nobel la première place dans le monde scientifique suédois de l'époque. Après un doctorat à Uppsala, il a voyagé à Paris, Berlin, Gottingen, collaborant avec Hermite, Weierstrass, Schering. Il a fondé au début des années 1880 la revue «Acta Mathematica», qui est toujours aujourd'hui l'une des trois ou quatre revues les plus prestigieuses en mathématiques au plan international.

Mittag-Leffler a su convaincre le roi Oscar de Suède et de Norvège (1829-1907) de soutenir financièrement la fondation d'Acta. Le roi, qui a été lui-même étudiant à Uppsala, est un mécène généreux pour l'activité scientifique. Mittag-Leffler propose donc au souverain de financer un prix qui célèbrerait son 60ème anniversaire.
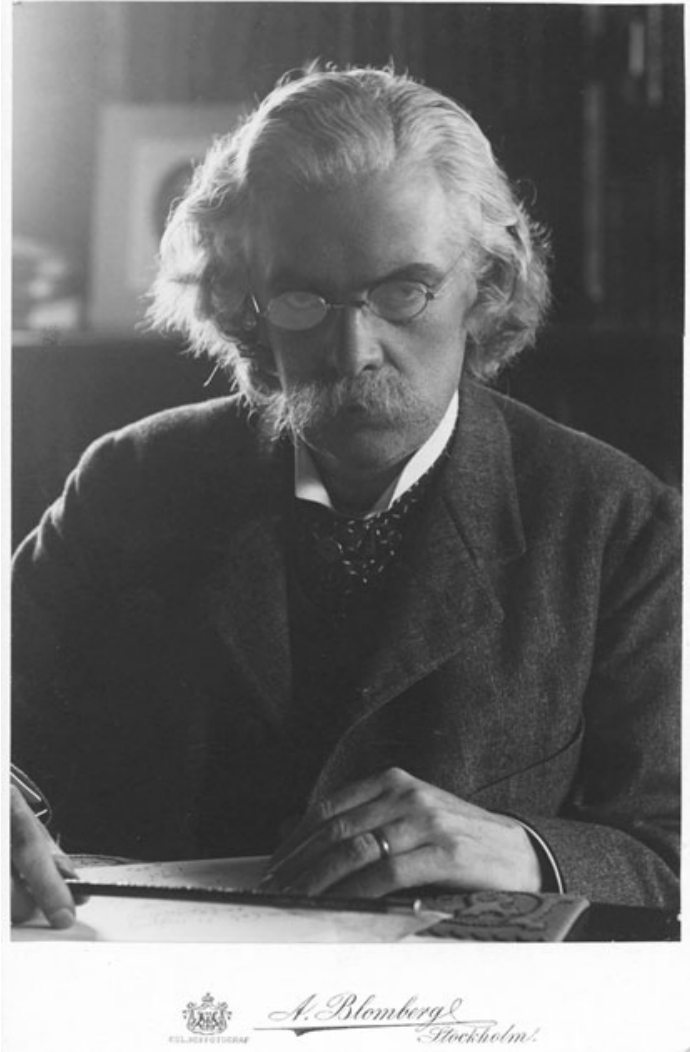

Fig. 2 Gösta Mittag-Leffler (1846-1927). Reproduced by generous permission of the LHSP-Archives Henri Poincaré. UMR 7117 CNRS/Université de Lorraine

Le jury est constitué de Mittag-Leffler lui-même, de Charles Hermite (Fig. 3) et de Karl Weierstrass (18151897). La prééminence de ces deux mathématiciens de la génération précédente au sein des écoles française et allemande garantit au prix une large audience.

L'annonce officielle est faite à la mi 1885 ; la date limite de soumission est fixée au 1er juin 1888. Le mémoire vainqueur sera publié dans les «Acta mathematica», et récompensé d'une médaille d'or accompagnée de 2,500 couronnes (Le salaire annuel de Mittag-Leffler est de 7,000 couronnes). Les candidats peuvent traiter l'un des 4 sujets proposés, ou un sujet libre de leur choix. Sur les 12 mémoires reçus, 6 se prévalent de cette possibilité, tandis que 5 se rattachent au premier sujet proposé, le problème des $n$ corps en mécanique céleste.

Hermite a contribué à la fondation des « Acta Mathematica ». Poincaré a été étudiant de Hermite, il a publié un article dans chacun des 5 premiers volumes de la revue. Il connaît Mittag-Leffler et n'a pas fait mystère de sa volonté de participer au concours. Malgré l'anonymat des soumissions, Mittag-Leffler n'a pas grand mal à identifier son collègue français comme l'auteur d'un mémoire qui se détache très nettement du lot. Ce mémoire, intitulé «Sur le 


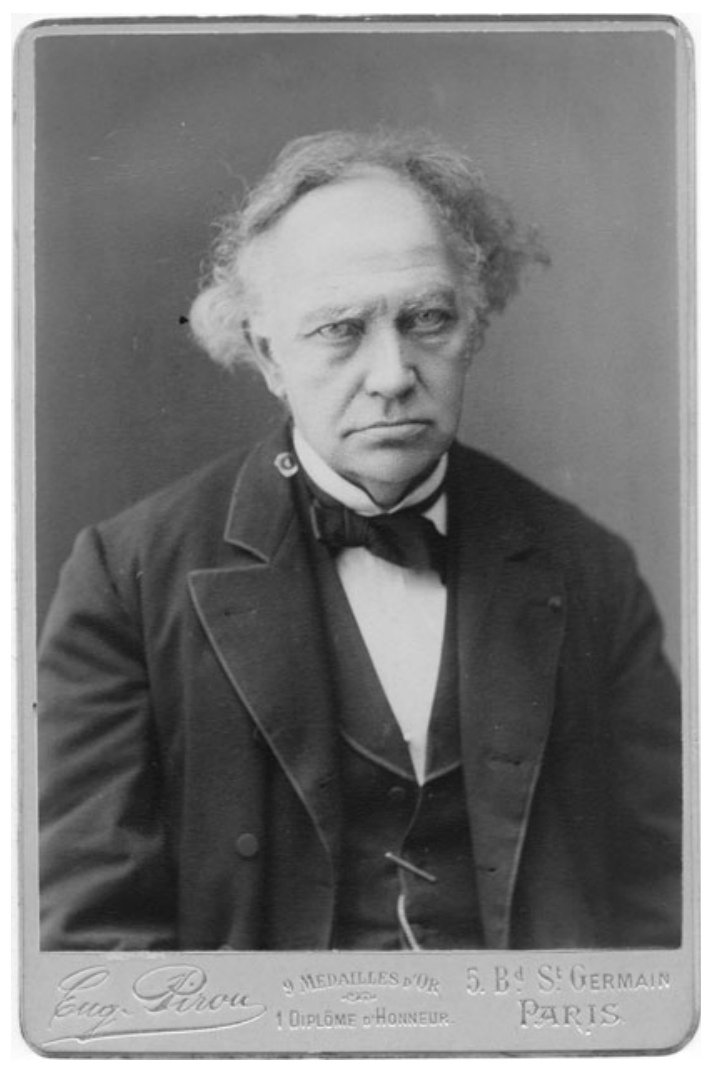

Fig. 3 Charles Hermite (1822-1901). Reproduced by generous permission of the LHSP-Archives Henri Poincaré. UMR 7117 CNRS/Université de Lorraine

problème des trois corps et les équations de la dynamique» fait très rapidement l'unanimité du jury. Le résultat est proclamé le 20 janvier 1889. L'autre mémoire distingué par le jury est l'oeuvre de Paul Appell et porte sur le développement en séries trigonométriques des fonctions abéliennes.

Le mémoire de Poincaré aurait dû être publié dans les « Acta mathematica » en octobre 1889. Il en sera autrement...

Lars Phragmen (1863-1937) est un jeune mathématicien suédois que Mittag-Leffler a chargé de la lecture détaillée des mémoires soumis. A la suite de ses commentaires, le mémoire de Poincaré, long de 160 pages initialement, s'est enrichi de 90 pages de notes supplémentaires. Vers juillet 1889, Mittag-Leffler transmet à Poincaré une demande d'éclaircissement de Phragmen. Poincaré s'aperçoit que les objections de Phragmen sont fondées, et découvre en reprenant le corps de son argumentation qu'il a commis une erreur sérieuse dans une autre partie du texte. Début décembre, il annonce à Mittag-Leffler que la rectification de l'erreur nécessite des changements substantiels dans son mémoire.

Craignant peut-être pour sa réputation scientifique, qui est moins établie que celle de Weierstrass, Hermite ou Poincaré lui-même, Mittag-Leffler récupère discrètement les quelques exemplaires du mémoire initial qu'il avait distribués à un cercle restreint de mathématiciens et astronomes. Il obtient de Poincaré que celui-ci règle les frais d'impression du mémoire initial, soit 3,500 couronnes, 1,000 de plus que le montant du prix. La version révisée, longue de 270 pages, est prête en avril 1890 et paraîtra dans les « Acta mathematica » en novembre 1890 [1].

Voilà pour les circonstances historiques, pour lesquelles le livre de June Barrow-Green cité en référence [2] m'a été précieux. Venons-en au contenu scientifique de l'épisode: je vais essayer d'expliquer l'erreur de Poincaré, la découverte à laquelle la rectification de cette erreur l'a mené, et le retentissement de cette découverte sur les mathématiques d'aujourd'hui.

Il faut d'emblée affirmer que même si l'on retranche au mémoire tout ce qui touche à l'erreur et à sa révision, le contenu en reste extraordinairement riche. Poincaré luimême en développera les idées dans les trois tomes des «Méthodes Nouvelles de la Mécanique Céleste», qui paraîtront entre 1892 et 1899 [3], et marqueront une refondation complète du domaine. On trouve aussi, dans la première partie du mémoire, ce qu'on appelle aujourd'hui le théorème de récurrence de Poincaré; ce résultat constitue l'acte fondateur de la théorie ergodique, branche cousine des systèmes dynamiques. Erreur ou pas, le prix était amplement mérité. Mais de tout ceci, je ne vais pas parler.

Un système dynamique, c'est un espace des phases avec une équation d'évolution; les points de l'espace des phases décrivent les états possibles du système considéré; l'équation d'évolution gouverne les changements d'états sur le court terme. Le but de la théorie est de comprendre l'évolution sur le long terme.

Souvent, un état peut-être déterminé par un nombre fini de paramètres et l'équation d'évolution est une équation différentielle décrivant la variation infinitésimale de ces paramètres. Poincaré, dès ses premiers travaux dans le domaine, va introduire un changement de point de vue fondamental. Ses prédécesseurs traitaient les équations différentielles comme des équations, et cherchaient à en représenter les solutions par des formules toujours plus sophistiquées. Poincaré va s'apercevoir que, pour la plupart des équations différentielles, on ne peut disposer d'aucune formule raisonnable. Il va traiter les équations différentielles comme des objets géométriques, une révolution conceptuelle qui ouvre des perspectives complètement inédites. C'est dans cet esprit que j'ai complètement évité les formules dans ce qui suit.

La mécanique céleste traite du mouvement des corps célestes-étoiles, planètes, satellites naturels ou artificiels, astéroïdes...-sous l'action de la gravitation classique, à l'exclusion de tous autres phénomènes physiques. La loi de gravitation universelle de Newton stipule que la force d'attraction mutuelle de deux corps est proportionnelle à 
chacune de leurs masses, et inversement proportionnelle au carré de leur distance.

Dans le problème des $n$ corps, les corps célestes sont assimilés à des masses ponctuelles sans diamètre. L'état du système est donc déterminé par les trois coordonnées de position et les trois coordonnées de vitesse de chacun des corps : l'espace des phases est de dimension égale à $6 n$; l'équation d'évolution est l'équation différentielle du second ordre qui traduit la loi de gravitation universelle.

Lorsqu'il y a seulement 2 corps, il n'est pas difficile de résoudre ces équations. Les solutions en ont en fait été découvertes par Kepler par l'observation céleste plus d'un siècle avant que Newton n'écrive ses équations. Chacun des corps parcourt une ellipse, le centre de masse occupant un des foyers de ces ellipses homothétiques; l'aire parcourue par le rayon joignant le centre de masse à l'un des corps est balayée à vitesse constante (on peut avoir aussi une hyperbole ou une parabole au lieu d'une ellipse, mais les corps s'échappent alors à l'infini).

La situation considérée par Poincaré dans son mémoire est le problème restreint des trois corps, le cas le plus simple après celui de deux corps. Dans ce problème restreint, on fait les hypothèses suivantes. On suppose d'abord que l'un des corps, appelons-le $m$, est de masse nulle. Il n'influence donc en rien le mouvement des deux autres corps, appelons-les $m_{1}$ et $m_{2}$, mais subit l'attraction gravitationnelle de ces corps. On suppose de plus que les corps $m_{1}$ et $m_{2}$, dont le mouvement doit obéir aux lois de Kepler, se déplacent à vitesse uniforme sur des cercles concentriques (dont le centre est le centre de gravité de ces deux corps). On cherche à comprendre la trajectoire du corps $m$, et on ne s'intéresse qu'aux trajectoires contenues dans le même plan que celles de $m_{1}$ et $m_{2}$. On suppose enfin que le rapport des masses de $m_{2}$ et $m_{1}$ est faible; on note $\mu$ ce petit paramètre.

Pour déterminer l'état du système, il faut connaître les deux coordonnées de position et les deux coordonnées de vitesse du corps $m$ dans le plan où se déroule le mouvement. L'espace des phases est donc de dimension 4. Le plus simple est en fait de se placer dans un repère tournant qui accompagne la rotation uniforme des corps $m_{1}$ et $m_{2}$. Dans ce repère, ces deux corps deviennent immobiles, ce qui simplifie l'écriture des forces de gravitation, mais introduit un terme correspondant à la force de Coriolis. Néanmoins, le système d'équations différentielles obtenu a la forme générale, dite hamiltonienne, associée à la plupart des systèmes d'origine mécanique; une conséquence fondamentale de cette propriété est la conservation au cours du temps d'une certaine fonction, le hamiltonien, calculable à partir de l'état du système. Cela veut dire que les solutions, qui sont des courbes dans l'espace des phases paramétrées par le temps, sont tracées sur les hypersurfaces (de dimension 3) représentant les différents niveaux possibles du hamiltonien (dans les situations classiques de la mécanique, le hamiltonien n'est rien d'autre que l'énergie totale du système).

Fixons le niveau du hamiltonien. Nous avons donc une hypersurface de dimension 3 sur laquelle sont tracées des courbes paramétrées par le temps. Dans cette hypersurface, Poincaré considère une surface $\Sigma$ (de dimension 2) transverse à la famille de courbes. Les équations d'évolution se traduisent par une transformation $T$ de cette surface $\Sigma$ dans elle-même : étant donné un point $x$ de $\Sigma$, on considère la courbe solution passant par $x$ à l'instant 0 et on désigne par $T(x)$ le premier point où cette courbe solution rencontre à nouveau $\Sigma$. Il s'agit donc à présent de comprendre les itérations successives de cette transformation $T$ de la surface $\Sigma$. On est passé d'une dynamique à temps continu en dimension 3 à une dynamique à temps discret en dimension 2.

Lorsque le paramètre $\mu$, rapport des masses de $m_{2}$ et $m_{l}$, est nul, il est facile d'analyser complètement la dynamique. Le corps $m_{l}$ est immobile à l'origine et le corps $m$, ne subissant pas l'attraction de $m_{2}$ décrit une ellipse (ou une hyperbole, ou une parabole; mais c'est le cas de l'ellipse qui nous intéresse dans la suite) dont l'origine est un foyer. Dans le repère tournant, cette ellipse présente un mouvement de rotation apparent traduisant la rotation uniforme de $m_{2}$ autour de l'origine. Il y a donc superposition de deux mouvements périodiques: la rotation du grand axe de l'ellipse (dans le repère tournant) à une vitesse angulaire uniforme et le déplacement sur l'ellipse du corps $m$ en balayant les aires à vitesse uniforme (deuxième loi de Kepler). Les périodes des deux mouvements sont indépendantes et en général incommensurables: le mouvement dans son ensemble n'est alors pas périodique. On dit que le système est complètement intégrable et que la dynamique correspondante est quasipériodique.

Pour la dynamique de la transformation $T$ sur la surface $\Sigma$, cela se traduit de la façon suivante. La surface $\Sigma$ est feuilletée par un système de courbes fermées; chacune de ces courbes est invariante par la transformation $T$; de plus, chacune de ces courbes peut être paramétrée par une coordonnée angulaire de façon que la transformation $T$ s'exprime comme une rotation dans cette coordonnée. L'angle de cette rotation dépend de la courbe considérée, et correspond au rapport des périodes des deux mouvements périodiques dans le cas du temps continu. Lorsque cet angle compté en nombre de tours, est un nombre rationnel, chaque point de la courbe est périodique sous l'action de $T$. Lorsqu'au contraire cet angle est irrationnel, et c'est le cas pour la plupart des courbes, les images successives d'un point de la courbe forment un ensemble dense dans la courbe.

Que se passe-t-il lorsque le paramètre $\mu$ n'est pas nul, mais simplement très petit? Dans quelle mesure va-t-on retrouver certains des aspects du cas $\mu=0$ ? Poincaré 
analyse d'abord le cas des orbites périodiques. Considérons pour fixer les idées le cas d'une courbe de $\Sigma$, invariante par $T$ lorsque $\mu=0$, pour laquelle l'angle de la rotation induite par $T$ s'annule. Tous les points de cette courbe sont donc fixés par $T$ lorsque $\mu=0$. Lorsque $\mu$ est petit mais non nul, Poincaré montre que seul un nombre fini de points (très voisins de cette courbe) sont encore fixés par $T$, et correspondent donc à des orbites périodiques pour le système en temps continu (dans le repère tournant).

Une analogie avec un système mécanique plus simple, le pendule, est utile. On considère le mouvement dans un plan vertical d'une barre rigide fixée à une de ses extrémités. En l'absence de pesanteur (correspondant au cas $\mu=0$ pour le problème restreint des 3 corps), on a un mouvement de rotation uniforme; en particulier toutes les positions sont des positions d'équilibre. Par contre, en présence de pesanteur, il n'y a plus que deux positions d'équilibre. La position verticale basse est un équilibre stable, la perturbation de cet équilibre conduit à de petites oscillations. La position verticale haute est un équilibre instable; si, à un temps infiniment lointain dans le passé, la barre s'éloigne de cet équilibre avec une vitesse infiniment petite, elle effectuera un tour complet pour revenir à l'équilibre à un temps infiniment lointain dans le futur avec une vitesse infiniment faible. Ce comportement remarquable est qualifié de doublement asymptotique par Poincaré; le vocabulaire moderne est homocline.

Revenons aux points fixés par la transformation $T$ sur la surface $\Sigma$. Poincaré montre que la moitié d'entre eux sont stables et l'autre moitié sont instables, au moins au niveau infinitésimal. Le passage de la stabilité infinitésimale à la stabilité locale ne sera obtenu que vers 1960 grâce aux succès de la théorie KAM (pour Kolmogoroff-ArnoldMoser); ce n'est pas ici notre sujet. Poincaré étudie de plus près les points fixes instables. Pour chacun de ces points fixes, Poincaré démontre qu'il existe une courbe remarquable tracée sur $\Sigma$ passant par ce point fixe, dite stable ou positivement asymptotique, caractérisée par la propriété suivante : quand on itère la transformation $T$ à partir d'un point de cette courbe, la suite de points obtenue ainsi converge vers le point fixe. Il existe de même une courbe, dite instable ou négativement asymptotique, caractérisée par la propriété duale : quand on itère l'inverse $T^{-1}$ de la transformation $T$ à partir d'un point de cette courbe, la suite de points obtenue ainsi converge vers le point fixe. Chacune de ces courbes est invariante sous l'action de la transformation $T$. Dans l'exemple du pendule pesant, les trajectoires homoclines associées à l'équilibre instable constituent à la fois la courbe stable et la courbe instable (il y a deux trajectoires homoclines suivant le sens de rotation du tour effectué).

Les courbes positivement et négativement asymptotiques des points fixes instables de la transformation $T$ coïncident-elles, comme c'est le cas pour le pendule pesant? Dans le cas du pendule pesant, outre un calcul direct, un argument de portée plus générale est le suivant: on a affaire à une dynamique en temps continu dans un espace des phases bidimensionnel; le théorème d'unicité des solutions d'équations différentielles garantit alors que les deux courbes asymptotiques sont égales dès qu'elles se rencontrent (en un point distinct du point fixe auquel elles sont associées).

Poincaré va chercher à déterminer la position de ces courbes positivement et négativement asymptotiques, en effectuant des développements par rapport aux puissances successives du petit paramètre $\mu$ (plus exactement, de la racine carrée de $\mu$ ). Dans la version initiale du mémoire, il montre que les deux courbes coïncident au premier ordre en $\sqrt{ } \mu$; il affirme aussi que les développements en les puissances successives de $\sqrt{ } \mu$ sont convergents. Dans la version corrigée du mémoire, il montre que les deux courbes coïncident à tous les ordres en $\sqrt{ } \mu$; si les développements étaient effectivement convergents, cela permettrait évidemment de conclure que les deux courbes sont égales. Hélas, la convergence, qu'il pensait être conséquence de principes généraux valables dans des situations similaires, n'a pas lieu : lui-même le montrera dans la version corrigée!

On peut penser que Poincaré, en rédigeant la version initiale du mémoire, avait vérifié que les deux courbes coïncident à tous les ordres en $\sqrt{ } \mu$ et donc (convaincu qu'il était alors de la convergence des développements) qu'elles étaient égales. Pour éviter le calcul délicat de ce développement à tous les ordres, il va chercher un raccourci en complétant le calcul (facile) au premier ordre en $\sqrt{ } \mu$ par un argument de nature topologique. A la base de cet argument se trouve la propriété que $T$ préserve les aires, propriété héritée de la nature hamiltonienne du système initial. L'argument montre effectivement que les deux courbes doivent se rencontrer (en un point distinct du point fixe instable). En temps continu, pour le pendule pesant, cela implique que les deux courbes coïncident. Mais pas en temps discret, comme c'est le cas pour la transformation $T$ !

En résumé, les arguments, grâce auxquels Poincaré pensait initialement pouvoir conclure que les courbes positivement et négativement asymptotiques coïncident, permettent seulement de prouver que ces courbes se rencontrent en des points distincts des points fixes auxquels elles sont associées. En général, en ces points d'intersection, les droites tangentes aux deux courbes sont distinctes; les trajectoires correspondantes sont dites homoclines transverses.

Quand on cherche, comme Poincaré lui-même l'a fait, à tracer dans toute leur extension des courbes positivement et négativement asymptotiques présentant des intersections homoclines transverses, on s'aperçoit rapidement que le fait que ces courbes soient invariantes par la transformation 
$T$ force une géométrie d'une complexité redoutable. Si Poincaré est bien conscient de cette complexité, il reviendra aux successeurs de Poincaré, George D. Birkhoff (1884-1944) et Steve Smale (né en 1930) de commencer à l'analyser.

Un des outils conceptuels fondamentaux, introduit par Alexandre Liapounov (1857-1918), est la mesure du taux de divergence (ou convergence) exponentielle des trajectoires au niveau infinitésimal. Pour le pendule pesant, cette divergence est toute entière concentrée au point d'équilibre instable. En présence de points d'intersections homoclines transverses, cette divergence exponentielle va se manifester pour toutes les trajectoires correspondant aux points d'intersection. C'est une telle divergence exponentielle qui caractérise les dynamiques de type chaotique qui sont à la base de l' «effet papillon».

Le fer à cheval de Smale est un modèle simplifié de la transformation $T$ où l'on est capable de décrire complètement le système d'intersections homoclines transverses associé à un point fixe instable. Un codage géométrique simple permet d'associer à chaque point d'intersection des courbes positivement et négativement asymptotiques une suite de 0 et de 1 (paramétrée par les entiers relatifs, et ne comportant qu'un nombre fini de 1). Inversement, toute suite de 0 et de 1 ayant ces propriétés est associée à un point d'intersection. La suite associée à un point à l'image $T(x)$ d'un point d'intersection $x$ est simplement la suite associée à $x$ décalée d'un cran vers la gauche. Quand on ne considère que la partie de la suite paramétrée par les entiers positifs ou nuls (cela revient à se concentrer sur l'évolution future en oubliant le passé), le passage de $x$ à $T(x)$ revient à multiplier par 2 le nombre dont la suite tronquée est le développement binaire : on retrouve la citation de Poe...

Malgré tous les progrès accomplis depuis une cinquantaine d'années dans notre analyse des systèmes dynamiques chaotiques (hyperboliques est le terme généralement utilisé par les mathématiciens), on aurait tort de croire que le problème restreint des 3 corps est aujourd'hui compris de façon satisfaisante. Une question centrale de la théorie des systèmes dynamiques, et qui est complètement ouverte à l'heure actuelle, est la suivante: choisissons au hasard un point de la surface $\Sigma$, et observons son orbite par les itérations successives de la transformation $T$. Y-a-t-il une probabilité non nulle (sur le choix du point initial) pour qu'on observe le long de cette orbite une divergence exponentielle des orbites au niveau infinitésimal ? La théorie KAM mentionnée auparavant nous garantit qu'à l'inverse il y a une probabilité non nulle de ne pas observer de divergence exponentielle car la dynamique de l'orbite sera de nature quasipériodique...

\section{Bibliographie}

1. Poincaré H.: Sur le problème des trois corps et les équations de la dynamique. Acta Math. 13, 1-270 (1890)

2. Barrow-Green.: Poincare and the three-body problem. History of mathematics, vol. 11. American Mathematical Society, Providence (1997)

3. Poincaré H.: Les méthodes nouvelles de la mécanique céleste, vol. 3. Gauthier-Villars, Paris (1892-1899)

\section{Author Biography}

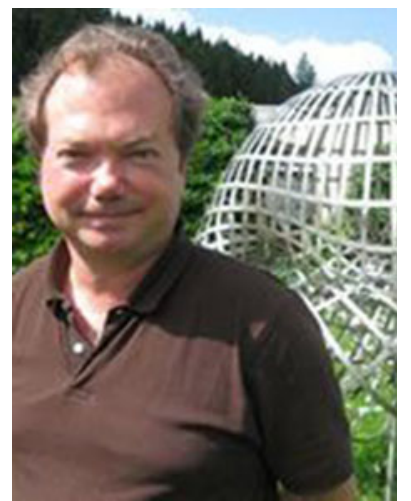

Jean-Christophe Yoccoz (1957) is a professor at the Collège de France. He was awarded the Fields Medal at the International Congress of Mathematicians in Zurich in 1994 for his work on the theory of dynamical systems. 